\title{
El impacto socioeconómico de la producción del
}

\section{maracuyá.}

The socio-economic impact of the production of the passion fruit.

Velasteguí López Efraín. ${ }^{1}$, Guamán Rodríguez Mónica. ${ }^{2}$

Resumen.

DOI: https://doi.org/10.33262/visionariodigital.v1i2.243

Es una planta que inicia su fructificación después la plantación. El periodo de vida es de 3 a 5 años esto puede variar. La productividad puede llegar a $70 \mathrm{Kg}$. de plantas siempre y cuando el cultivo de manera silvestre y manejo adecuado del suelo. El llega al estado de madurez cuando se desprende del fruto y cae al suelo es por eso que se recomiendo recolectar después de 34 días aproximadamente.

Es de suma importancia de contar con suelos profundos, bien drenados, de textura franca, con buena capacidad para poder retener la humedad. Crece mejor en climas cálidos y templados, aunque retrasen el inicio de producción.

La pasiflora es una planta que sé que cultiva en suelos profundos es por eso que los usos de semillas deben ser cuidadosamente seleccionadas para su alta productividad, como es en el caso del maracuyá amarillo. Generalmente estas semillas se germinan en bolsas plásticas y luego son llevadas al campo cuando tenga una medida aproximada de $25 \mathrm{~cm}$. Su plantación debe estar distanciada entre filas y entre plantas, se calcula que por lo menos en una hectárea puede llegar a tener 1,000 plantas. Esta plantación se manejará con tutores y espaldera simple con dos filas de alambre, utilizando para ello alambres, postes para el soporte y tutores para guiar los brotes.

La fructificación o cosecha se empieza a partir de 7 a 10 meses después de su plantación y el fruto llega a su madurez cuando se desprende de la planta y cae al suelo.

Palabras claves: Socioeconómico, Producción, Maracuyá, Impacto.

\section{Abstract}

It is a plant that begins its fruiting after the plantation. The period of life is of 3 to 5 years this can vary. The productivity can reach $70 \mathrm{Kg}$. Of plants as long as the cultivation of

\footnotetext{
${ }^{1}$ Universidad Técnica de Ambato, Ambato, Ecuador, le.velastegui@uta.edu.ec ${ }^{2}$ Universidad Técnica de Cotopaxi Extensión la Maná. Ecuador, monyguamansdeyop@gmail.com 
wild way and adequate management of the soil. It reaches the state of maturity when it falls off the fruit and falls to the ground that is why it is recommended to collect after approximately 34 days.

It is very important to have deep soils, well drained, with a clear texture, with good capacity to retain moisture. It grows best in warm and temperate climates although they delay the start of production.

The passionflower is a plant that is grown in deep soils that is why the use of seeds should be carefully selected for high productivity, as in the case of yellow passion fruit. Generally these seeds are germinated in plastic bags and then taken to the field when it has a measure of approximately $25 \mathrm{~cm}$.

Its plantation must be separated between rows and between plants, it is estimated that at least one hectare can have 1,000 plants. This plantation will be managed with tutors and simple trellis with two rows of wire, using wires, poles for the support and tutors to guide the shoots.

Fruiting or harvesting starts from 7 to 10 months after planting and the fruit reaches maturity when it falls off the plant and falls to the ground.

Keywords: Socioeconomic, Production, Passion fruit, Impact.

\section{Introducción.}

El maracuyá es una fruta tropical o también llamada fruta de la pasión o parchita y denominada la fruta de la pasión, de un sabor un poco ácido y con aroma. Las variedades varían en el tamaño color y sabor. Actualmente 40 países utilizan el maracuyá en el campo comercial para satisfacer la demanda interna y solo en nuestro país se han cultivado la maracuyá amarilla y púrpura debido a la inestabilidad de los precios y apoyo del gobierno. El cultivo de maracuyá es una planta que se ha convertido en un fruto de alto consumo, permitiendo aumentar la demanda. Es por esto la importancia de mejorar la producción, en toneladas por hectárea, el tamaño del fruto, peso, los grados brix, la precocidad y la resistencia al virus causante de la malformación, conocido como el virus de noni.

Es una planta que de acuerdo a las condiciones fitosanitarias se produce desde los 300 msnm hasta los $1000 \mathrm{msnm}$, el clima en que se desarrolla requiere que hay alternancia de época de humedad y seca y de 1000 a 1500 milímetros d precipitación, hace de la familia de las pasifloras y su nombre científico es: passiflora edulis v. flavicarpa. Es originaria de la región amazónica del Brasil, la familia comprende doce géneros con aproximadamente 500 especies distribuidas en américa, Asia y áfrica.

Según (Arroyave , 2014., Delfin, 2016., Elgeta, 2014., Fernandez, 2014) Afirma que: El desarrollo de las pequeñas y medianas empresas (Pymes) en el mercado global es una prioridad para el crecimiento económico de cada país. Si las Pymes quieren mantenerse, crecer y desarrollarse en un entorno mundial y dinámico tienen que plantear estrategias que les permitan alcanzar su desarrollo empresarial. En este artículo se explican ampliamente las diferentes definiciones que incluyen el desarrollo empresarial como 
concepto integrador, como son el crecimiento económico, la cultura empresarial, el liderazgo, la gestión del

conocimiento y la innovación. Se pretende con ello que el empresario identifique la necesidad de capacitarse en estos conceptos, actuales y detonantes de competitividad.

El desarrollo es un concepto histórico que ha ido evolucionando, por lo que no tiene una definición única. Aun así, se puede decir que desarrollar es el proceso por el cual una comunidad progresa y crece económica, social, cultural o políticamente.

Desde sus inicios, las teorías relacionadas con el desarrollo se interesaron por los procesos de enriquecimiento material, es decir, por el incremento del volumen de producción de bienes y servicios. Estas teorías economicistas entendían que el medio para alcanzar el desarrollo era la acumulación de capital físico. Se defendía que un aumento del producto interior bruto per capita reduciría la pobreza e incrementaría el bienestar de la población. Esta premisa se basaba en que, a más producción, más renta, y, a más renta, mayor bienestar económico. Es decir, que el desarrollo estaba directamente relacionado con el crecimiento económico, tanto de los países como de las personas. Como apunta Keith Griffin en su ensayo "Desarrollo humano: origen, evolución e impacto", el crecimiento se convertía no sólo en el medio para alcanzar el desarrollo, sino en el fin del desarrollo mismo.

\section{Criterio Personal}

El desarrollo, como objeto de estudio, requiere para su abordaje de una pluralidad teóricometodológica. En este sentido, la visibilización de la emergencia de las nuevas perspectivas de este, puede conducir no solo a comprender la complejidad de su producción como noción y práctica, sino que, además, nos exige el diseño de instrumentos complejos para su aprehensión y también para su ponderación. En términos generales, cualquiera que sea la perspectiva, se asume explícita o implícitamente que reconocer al desarrollo como un fenómeno multidimensional (social, económico, político, cultural, ambiental) implica la necesidad de analizarlo desde la inter y la transdisciplinariedad y, en términos específicos de la medición, a la necesidad de establecer un sistema integrado de indicadores sin fines de ordenación (lista de países), que tenga en cuenta la complejidad de los territorios y que no se base en la aplicación acrítica de dispositivos impuestos por los organismos de financiamiento externo.

\section{Impacto}

Según (Iturbe, 2015., Jaramillo , 2015., Marin , 2015., Mayorga , 2015)Afirman que: El término "Economía" proviene del griego, en concreto, de la conjunción de dos voces: "oikos" que significa casa (en el sentido de bienes, patrimonio) y "nomos" que significa administrar. En la actualidad, su significado mantiene en cierta forma el original, ya que la Economía es la ciencia que estudia el mejor modo de utilizar unos recursos que son escasos para satisfacer las necesidades de la sociedad. 
Sin embargo, a pesar de que los fenómenos económicos son tan antiguos como el hombre, la Economía como ciencia es relativamente joven. Se considera que nació con la obra del economista escocés Adam Smith (1723-1790), La riqueza de las naciones, publicada por

primera vez en 1776, donde sistematizó el conjunto de conocimientos que integraban hasta ese momento la Economía.

Así, este autor, dentro de sus múltiples contribuciones a la economía, lo hizo en el campo de la teoría del comercio internacional, al haber desarrollado el principio de la ventaja comparativa, a través de la cual explica la especialización que buscan los países para mejorar sus relaciones comerciales. En sus investigaciones utilizó los métodos deductivo y abstracto

Los distintos agentes económicos, cómo influyen unos sobre otros y el resultado final de esas influencias mutuas. Para simplificar esta presentación, tomaremos a personas y familias como un solo conjunto, y excluiremos toda relación con otros países. Más adelante examinaremos estas relaciones al hablar del comercio exterior, es decir, de lo que el país compra y vende a otros países. Las familias necesitan ganar ingresos, para lo cual venden su trabajo a las empresas y al Gobierno.

Gráfico 1. Inteligencia de mercado del maracuya.
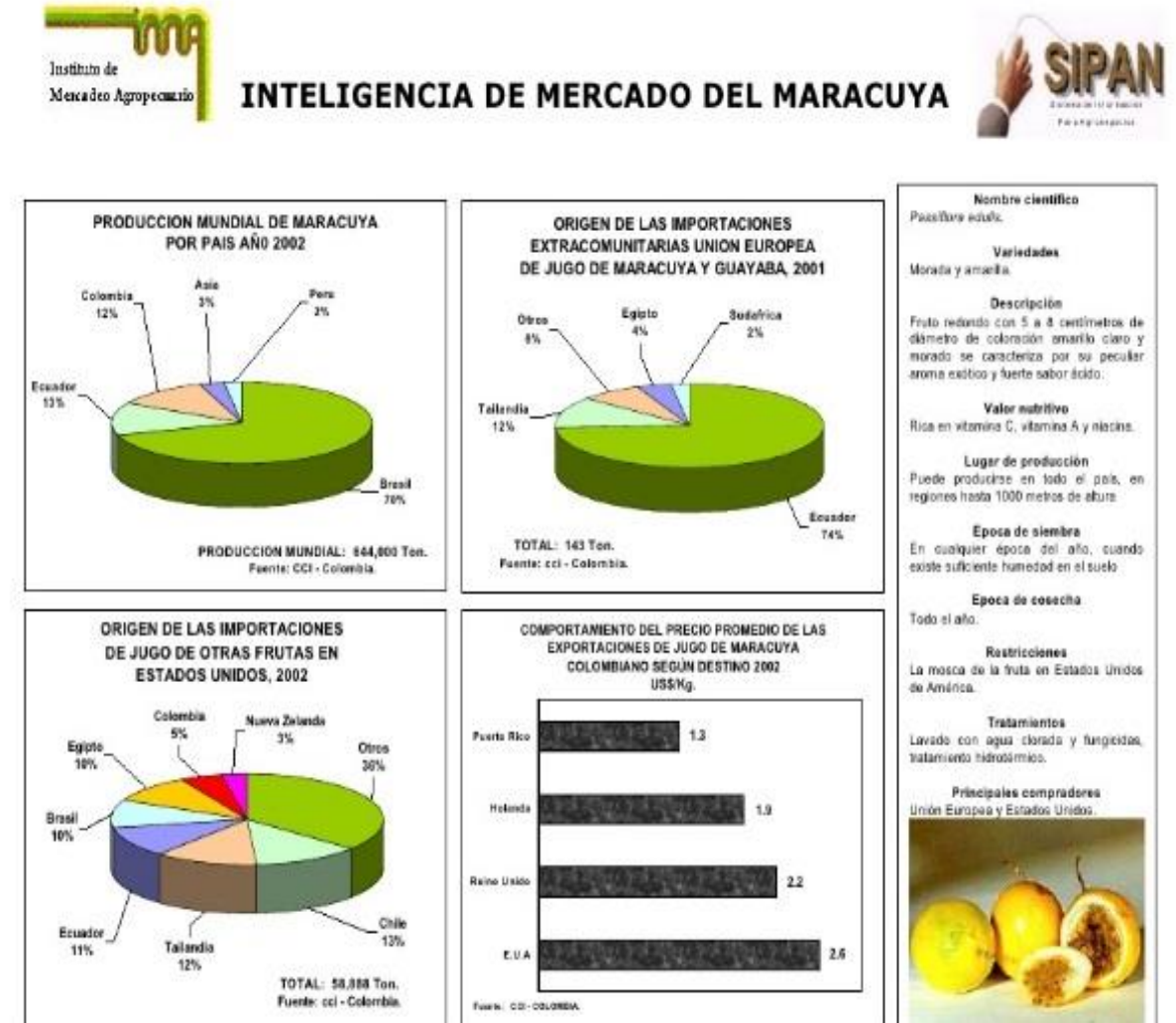
Autor: Luis Alejandro Calderón.

Fuente: https://es.slideshare.net/janoac/analisis-del-maracuya-inteligencia

También necesitan satisfacer sus necesidades, para lo cual deben adquirir bienes y servicios que compran a las empresas. Además, deben pagar impuestos al Gobierno a cambio de los

servicios que este presta. Por otra parte, las familias (algunas de ellas), son dueñas de las empresas, y reciben las utilidades (o pagan las pérdidas) que las empresas generen. Por su parte, las empresas necesitan comprar trabajo a las familias para poder producir, a la vez que venden sus productos a estas y al Gobierno.

Al igual que las familias, pagan impuestos al Gobierno a cambio de los servicios que este proporciona. Las utilidades que obtengan como resultado de sus ingresos por ventas menos sus costos, luego de pagar los impuestos, las entregan a sus dueños, algunas familias.

La producción de calzado en el Ecuador es una rama con perspectiva al desarrollo de la productividad del país, este sector industrial es muy diversificada, presenta además una gran variedad de productos para el mercado internacional. El uso de la tecnología, la innovación tecnológica y el mejoramiento de la calidad son factores que contribuyen a mejorar la competitividad de las MIPYMES, sin embargo, en el Ecuador sólo el 30\% de las mismas utilizan las ventajas tecnológicas de la información y comunicación (TIC), cifra muy baja en relación al 50\% de los demás países de América Latina, siendo Costa Rica, Argentina y Chile los países que más utilizan estos sistemas en la región, según la Red Global de

\section{Exportación. \\ Criterio Personal.}

Por otro lado, el $45 \%$ de empresas utilizan el servicio de internet, el $87 \%$ han realizado algún tipo de innovación, ya sea de productos o de procesos y, apenas el 5\% tiene algún tipo de certificación de calidad, según la Encuesta Nacional de Micro, Pequeñas y Medianas empresas de la industria manufacturera, 2007 (Jácome y Kimg 2013). De acuerdo a los resultados de las Cuentas Nacionales Trimestrales publicados por el Banco Central del Ecuador (BCE), el Producto Interno Bruto (PIB) tuvo un crecimiento anual de $4.5 \%$ en el año 2013 con respecto a 2012, ubicando al país como una de las economías con mejores resultados en la región.

Resulta bastante complicado el encontrar una definición única de la ciencia económica y en consecuencia su objeto de estudio puede aparecer impreciso. Lo afirmado obedece a que tanto los padres de la economía como los economistas de las recientes escuelas de pensamiento no se han puesto de acuerdo sobre la temática debido a los diferentes enfoques teóricos que caracterizan a cada una de las escuelas de pensamiento. Por ejemplo, Gary Becker2 en su obra "El enfoque económico de la conducta humana" 
plantea que "La definición de Economía en términos de bienes materiales es la más limitada y menos satisfactoria.

Con este propósito desarrolló la teoría del valor trabajo. Se preocupó también de averiguar las causas del crecimiento o, si se prefiere, del origen de la riqueza de los países a través del análisis de los factores que explican la distribución de la renta.

\section{Maracuyá.}

Según (Pereira, 2015., Perez, 2015., Pozo, 2013., Sañudo, 2015., Vizuete, 2015) Afirman que: La producción sirve para acercar un bien o servicio y que éste pueda satisfacer las

necesidades de los consumidores. Por ello, las actividades de la producción son la fabricación, el transporte, el almacenamiento y la comercialización.

Si en el caso que venimos considerando, el helado que la empresa lanza al mercado experimenta una demanda creciente, ésta deseará expandir la producción. De forma inmediata la empresa puede hacer que la mano de obra existente trabaje horas extraordinaria, y también puede incrementar el número de empleados contratados. A largo plazo, y si continúa la presión de la demanda, los gerentes de la empresa se plantearán la conveniencia de ampliar las instalaciones e incluso de construir una nueva fábrica.

La producción es el proceso mediante el cual la empresa transforma un conjunto de factores de producción en un producto cuyo valor debe ser mayor que la suma de los valores de los factores utilizados (lógicamente, si el valor fuese igual o menor, la actividad de la empresa no tendría ningún sentido). Hay diferentes maneras de organizar la producción. Pensemos por ejemplo en una empresa constructora, cuyos productos son los edificios que construye.

Los principales factores de producción son la maquinaria utilizada, los materiales de construcción y el trabajo de arquitectos, técnicos, operarios, etc. La empresa puede organizar su producción de dos maneras: puede diseñar un modelo de edificio y construir siempre el mismo modelo, con variaciones mínimas.

Esto es lo que llamaríamos un sistema de "producción rígida". Alternativamente, la empresa puede diseñar cada edificio prácticamente "desde cero", haciendo en cada caso un estudio de la localización, el uso que se va a dar al edificio, etc. En este caso, se trataría de un sistema de "producción flexible".

os sistemas productivos agropecuarios se deben enfocar hacia la productividad, competitividad y calidad. Su orientación está basada en la normatividad vigente de cada país, para el caso de Colombia, la Norma NTC 5400 de 2005 de Buenas Prácticas Agrícolas - BPA y Resolución 4174 de 2009. Con esto se asegura la inocuidad, protección del ambiente y condiciones laborales favorables para el personal que participa de la actividad agrícola.

Es de gran interés la promoción de estas prácticas por cuanto conforman un aporte al bien público y posibilitan mejores niveles de competitividad para la agricultura, además ayudan a la divulgación y mantenimiento de la confianza de los productos del país. Las Buenas Prácticas Agrícolas se deben adoptar y aplicar desde la selección del material 
vegetal hasta la actividad de cosecha y poscosecha, permitiendo la trazabilidad de la actividad productiva y la garantía para el consumidor final. La necesidad de aplicar las BPA en el cultivo de Maracuyá se justifica por la necesidad generada de llevar a cabo procesos amigables con el ambiente.

Según la Corporación Colombia Internacional (CCI), en el año 2002 la producción mundial de maracuyá fue de 640.000 toneladas, siendo Brasil el mayor productor representando un 70\%; le siguen Ecuador y Colombia con un 13\% y $12 \%$ respectivamente. Cabe destacar que en ese año la producción disminuyó aproximadamente en $18 \%$ respecto al 2001, debido a la caída de la producción de Ecuador, que había tenido incrementos notorios en la productividad en los últimos años. Sin embargo, el consumo de frutas como el maracuyá se ha incrementado de manera notoria en países desarrollados. Este factor ha llevado a una

demanda de maracuyá en el comercio internacional, buscando tener disponibilidad de este frutal durante todo el año.

Gráfico 2. Producción de maracuyá.

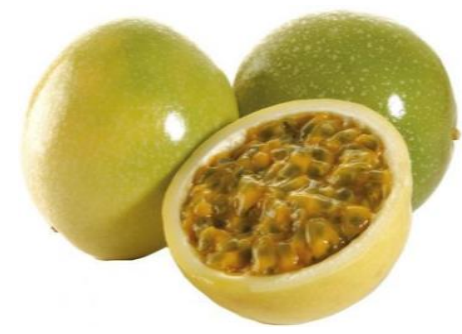

Autor: Cesar Aching Guzmán.

Fuente: https://puntodevistaypropuesta.wordpress.com/2015/02/24/

Para la definición de la agenda de desarrollo de este estudio, se empleó una metodología propuesta por el Ministerio de Agricultura y Desarrollo Rural, que ha sido una directriz común para las cadenas que vienen participando en este proceso, con el propósito de facilitar la toma de decisiones de inversión y direccionamiento.Según el Ministerio de Agricultura y su observatorio de SCNTA en marzo del año 2013, se establecen la vigilancia tecnológica y el análisis de las tendencias de investigación como una práctica internacional de obligatorio cumplimiento para el caso de las actividades de $\mathrm{I}+\mathrm{D}$, donde el conocimiento del entorno tecnológico propende por sustentar y justificar la inversión de recursos humanos y materiales en distintos proyectos de investigación.

Teniendo en cuenta la importancia del sector agrícola y la necesidad de generar productos con mayor valor agregado, la vigilancia tecnológica surge con el objetivo de identificar oportunidades y limitaciones que permitan dar respuesta con procesos de innovación enfocándose en las tecnologías disponibles o que acaban de aparecer, capaces de intervenir en nuevos productos o procesos.

El enfoque del proceso de vigilancia tecnológica tiene como objeto identificar las tendencias, entidades, investigadores, países y enfoques específicos para las demandas 
asociadas a los productos en estudio, teniendo como prelación aquellas relacionadas directamente con desarrollos tecnológicos que involucren productos, subproductos y procesos, relacionados con la investigación básica desarrollada en universidades, instituciones de CyT y centros de investigación teniendo como productos de valoración y cuantificación la producción científica en términos de artículos en revistas indexadas.

\section{Criterio Personal}

Es factible alimentar a las plantas vía foliar particularmente para corregir deficiencias de elementos menores y en el caso de elementos mayores, $\mathrm{N}-\mathrm{P}-\mathrm{K}$, es necesario recalcar que el abonamientos foliar solamente puede ser complementario y en ningún caso puede sustituir la fertilización al suelo; debido a que las dosis de aplicación por vía foliar son muy pequeños en relación a las exigencias del cultivo.

La parchita o maracuyá (Passiflora edulis f flavicarpa Degener) es un rubro frutícola que ha experimentado un creciente aumento del área de cultivada, por lo que se hace necesario el desarrollo de actividades agroeconómicas que posean ventajas comparativas para la exportación y constituyan fuente de materia prima para la agroindustria local. Por ser un cultivo que se propaga generalmente por semilla y que puede explotarse en forma comercial, el incremento de la superficie cultivada amerita la obtención de plantas sanas, vigorosas, de tamaño uniforme y en cantidades suficientes para satisfacer la demanda, que garanticen el éxito de la fundación o cultivo de este frutal.

Entre los problemas que afectan la producción de parchita o maracuyá se destacan: inexistencia de material adecuado de propagación, el complejo de enfermedades, manejo empírico de las densidades de plantación, aplicación inadecuada falta de normas de calidad y precario manejo de la cosecha y poscosecha. Todos estos problemas originan bajas producciones y problemas de rentabilidad.

Gráfico 3 .Niveles de Producción en el aspecto Mundial

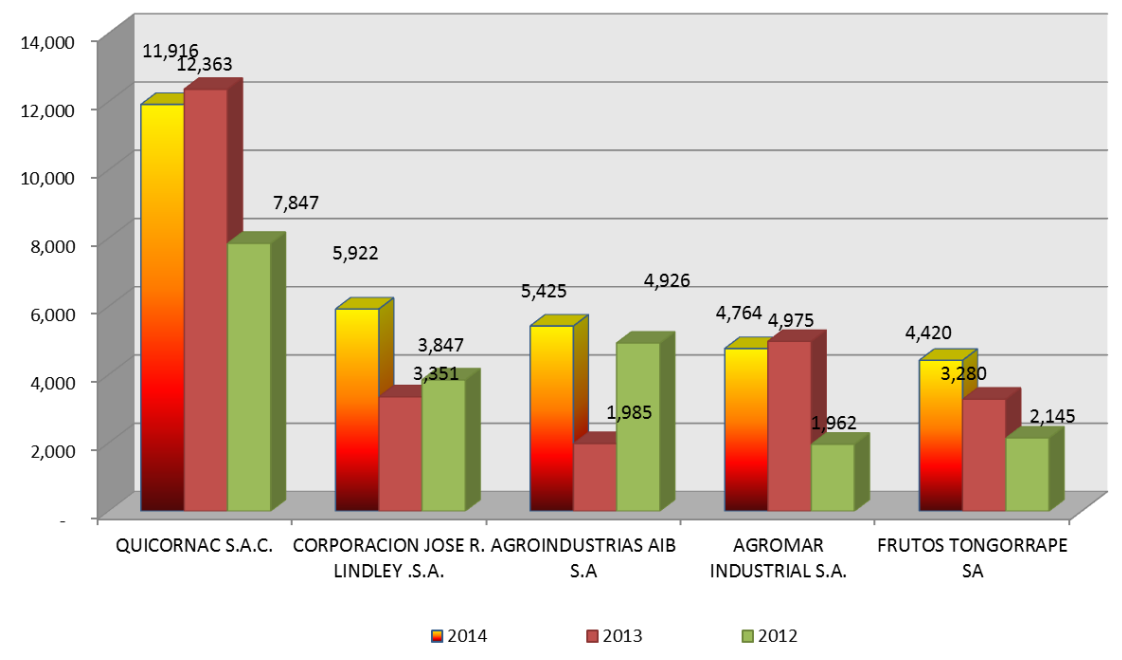

Autor:Wilfredo Koo. 
Fuente: https://www.agrodataperu.com/2014/12/maracuya-jugos-perunoviembre-2014.html

\section{Conclusiones.}

- Se puede decir que la producción de maracuyá se caracteriza por ser una actividad que ha generado ingreso a la Economía Nacional.

- El maracuyá es una buena opción para producir, puesto que sus derivados han permitido que nuevos productos cumplan con parámetros de calidad y permita satisfacer las necesidades del consumidor.

- Según análisis se ha establecido que Estados Unidos y la Unión Europea en especial Alemania son los países más adecuados para exportar éste producto.

- La producción de maracuyá colombiana tiene gran oportunidad de crecer favorablemente frente a otros países y de ofrecer diversos productos descendientes de ésta.

\section{Referencias.}

Arroyave , I. (2014). Manual del cultivo de Maracuyá bajo. Recuperado el 15 de 10 de 2018,

Delfin, F. L. (2016). Importancia y análisis del desarrollo empresarial. Obtenido de http://www.scielo.org.co/pdf/pege/n40/n40a08.pdf

Elgeta, C. (2014). Economia para todos. Recuperado el 15 de 10 de 2018, de https://www.economiamascerca.cl/massad/docs/economia_para_todos.pdf

Fernandez, J. (2014). La producción y la empresa. Recuperado el 15 de 10 de 2018, de https://www.mheducation.es/bcv/guide/capitulo/8448149971.pdf

Iturbe, A. (2015). SISTEMAS DE PRODUCCIÓN. Recuperado el 15 de 10 de 2018, de https://www.google.com.ec/search?q=sutores\&rlz=1C1CHBD_esEC806EC806\&oq=su tores\&aqs=chrome..69i57j015.2304j0j7\&sourceid=chrome\&ie=UTF-8

Jaramillo , E. (2015). El cultivo del Maracuyá en Ecuador. Recuperado el 15 de 10 de 2018 ,

https://www.researchgate.net/publication/312536029_El_cultivo_del_Maracuya_en_Ec uador

Marin , N. (2015). Red TecnoParque Colombia. Recuperado el 15 de 10 de 2018, de http://www.camaramedellin.com.co/site/Portals/0/Documentos/2016/competitividad/Inf orme\%20final\%20VT\%20Maracuy\%C3\%A1.pdf

Mayorga , C. (2015). I Congreso Iberoamericano de Investigación sobre MIPyME. $\begin{array}{lllllll}\text { Recuperado el } & 15 & \text { de } & 10 & \text { de }\end{array}$ http://portal.uasb.edu.ec/UserFiles/385/File/Los\%20procesos\%20de\%20produccion\%20 y\%20la\%20productividad\%20en\%20la\%20industria\%20de\%20calzado.pdf 
Pereira, V. (2015). ESTUDIO A LA APLICACIÓN DE TRES FRECUENCIAS Y DOS DOSIS DE N-P-K MÁS UNA FÓRMULA DE FERTILIZANTE FOLIAR EN EL CULTIVO DE MARACUYÁ. Recuperado el 15 de 10 de 2018, de http://repositorio.ug.edu.ec/bitstream/redug/7384/1/TESIS\%20DE\%20GRADO.pdf Perez , J. (2015). EOM. Recuperado el 15 de 10 de 2018, de https://elordenmundial.com/introduccion-al-concepto-de-desarrollo/

Pozo, R. (2013). Conceptos y principios de economía y metodologías utilizadas en la investigación económica. TENDENCIAS, 232.

Sañudo, M. (2015). DESARROLLO Prácticas y discursos emergentes en América Latina. $\begin{array}{lllllll}\text { Recuperado el } & 15 & \text { de } & 10 & \text { de } & 2018, & \text { de }\end{array}$ http://biblioteca.clacso.edu.ar/clacso/gt/20160309021832/Desarrollo_practicas.pdf Vizuete , F. (2015). La razón de ser de la Economía. Recuperado el 15 de 10 de 2018, de http://econopilar.com/wp-content/uploads/2015/09/Tema-01-La-economia-comociencia.pdf

\section{Para citar el artículo indexado.}

Velasteguí E, Guaman M. (2018El Impacto Socioeconómico de la Producción de la Maracuya Revista electrónica Visionario Digital 1(1), 65-74. Recuperado desde: http://cienciadigital.org/revistacienciadigital2/index.php/VisionarioDigital/article/view/ $\underline{243 / 543}$

\section{Ciencia}

El artículo que se publica es de exclusiva responsabilidad de los autores y no necesariamente reflejan el pensamiento de la Revista Ciencia Digital.

El articulo queda en propiedad de la revista y, por tanto, su publicación parcial y/o total en otro medio tiene que ser autorizado por el director de la Revista Ciencia Digital.
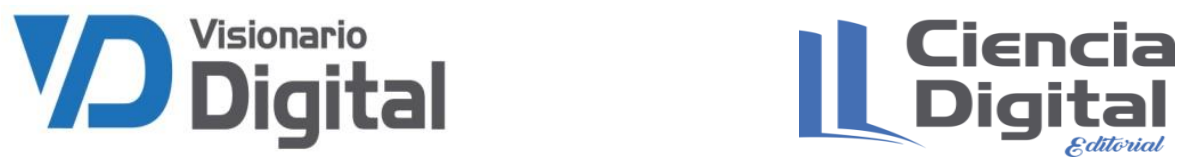